\title{
Interactions between Rotation and Pulsation
}

\author{
Rich Townsend \\ Department of Physics $\&$ Astronomy, University College London, \\ Gower Street, London WC1E 6BT, UK
}

\begin{abstract}
In this contribution, I will examine the interaction between stellar rotation and pulsation. I begin with a brief review of the non-rotating case, emphasizing the character of pulsations as azimuthally-propagating waves. I then go on to discuss how these waves are modified under the influence of the centrifugal and Coriolis forces. Through simple arguments, I outline the conditions under which each force can become significant in determining the wave dynamics. Particular attention is paid to the Coriolis force, since it is responsible for the formation of a waveguide, which confines the pulsation to a narrow band centered on the stellar equator. Using the example of a prograde sectoral pulsation mode, I explain the basic physical principles underlying this trapping.

The Coriolis force is also responsible for the existence of Rossby waves, which are not found in non-rotating stars. I demonstrate how these waves may be understood in terms of a conservation law for angular momentum, and review their most important characteristics. I then examine how rotation modifies the frequencies of pulsation, and explain how observations of such modifications can provide information regarding a star's rotation rate. To conclude, I focus on the converse of the pulsation-rotation interaction: how the transport of angular momentum by pulsation might be important in determining the evolution of a star's rotation profile.
\end{abstract}

\section{Introduction}

Within a stellar astrophysical context, the phenomena of rotation and pulsation each constitute entire fields of study in their own right. Therefore, it is not surprising that the interaction between the two is complex, and that studies of this interaction - often accompanied by many pages of daunting equations are doubly so. Of course, such 'formalism overdrive' is entirely necessary when we wish to undertake detailed studies; however, for those working outside the pulsation-rotation niche, it acts as a formidable barrier against an appreciation of the fundamental physics underpinning the problem.

Fortunately, the pulsation-rotation interaction is one of those rare instances where the Devil is not in the details: a reasonably-comprehensive grasp of the subject can be obtained from simple physical principles. This I hope to convey in the present paper, where I review at a general level the manner in which stellar rotation influences stellar pulsation, and vice-versa. For those wanting a more in-depth review of the topic, the excellent monograph by Unno et al. (1989) is a good place to start, although I should remark that some parts of their analysis have been superseded by more-recent studies. 


\section{Background}

To provide the necessary background information, a brief review of the phenomenon of pulsation in non-rotating stars is appropriate. In a nutshell, stellar pulsation is the excitation of one or more of the global oscillation modes of a star, which results in periodic perturbations to the position, velocity and physical state of every constituent fluid element. The excitation arises due to the existence of some mechanism, which draws energy from a source and supplies it to the pulsation; the source varies from star to star, and can be either internal (e.g. the local radiation field within the star - see Dziembowski \& Pamyatnykh 1993), or external (e.g. the torque generated by a binary companion - see both Koenigsberger and Rieutord, these proceedings).

Irrespective of the excitation mechanism, however, the spatial and temporal behaviour of the perturbations due to an individual pulsation mode may always be described by expressions of the form

$$
\Delta y(r, \theta, \phi, t)=f_{y}(r, \theta) \sin (m \phi+\omega t) .
$$

Here, $\Delta y(r, \theta, \phi, t)$ represents the perturbation to some generic quantity $y-$ be it temperature, pressure, velocity, etc. - at spherical-polar co-ordinates $\{r, \theta, \phi\}$ and time $t$. The function $f_{y}(r, \theta)$ describes the radial and polar dependence of perturbations; its precise form (see, e.g. Unno et al. 1989) is not important here. What is important, however, is the sinusoidal term on the right-hand side of the equation, describing wave motion in the azimuthal direction with angular frequency $\omega$ and angular wavelength $1 / \mathrm{m}$. The phase velocity of the wave is given by $c=-\omega / m$; therefore, if we adopt the convention that $\omega$ always be positive, it is clear that positive- $m$ modes correspond to propagation in the negative- $\phi$ (i.e., retrograde) direction, and negative- $m$ modes to propagation in the positive- $\phi$ (i.e., prograde) direction.

Since the star is invariant under a $360^{\circ}$ axial rotation, the 'azimuthal order' $m$ must be an integer, ensuring that $\Delta y$ (cf. Eq. 1) is single-valued. Likewise, since the radius of a star is finite, the pulsation frequency $\omega$ is restricted to a discrete spectrum of values, much like the case of waves on a string clamped at both ends. An important fiducial point in this spectrum is the 'dynamical frequency'

$$
\omega_{\mathrm{d}}=\sqrt{\frac{G M}{R^{3}}},
$$

which depends on the mass $M$ and radius $R$ of the star; here, $G$ is the constant of gravitation. Modes with $\omega>\omega_{d}$ have the character of acoustic waves, where the restoring force on displaced fluid elements arises from local pressure variations. Such p (pressure) modes are characterized by fluid motions predominantly in the radial direction. In contrast, modes with $\omega<\omega_{d}$ behave more like water waves, where the restoring force is the buoyancy arising from gravitational stratification. The fluid motions associated with these g (gravity) modes are predominantly in the tangential (i.e., azimuthal and polar) directions.

To illustrate the foregoing discussion, Fig. 1 shows the effects on a star of two $m=-4$ modes, identical in all aspects save for their frequencies: one has $\omega=$ $10 \omega_{d}$, corresponding to a p mode, and the other $\omega=0.1 \omega_{d}$, corresponding to a 

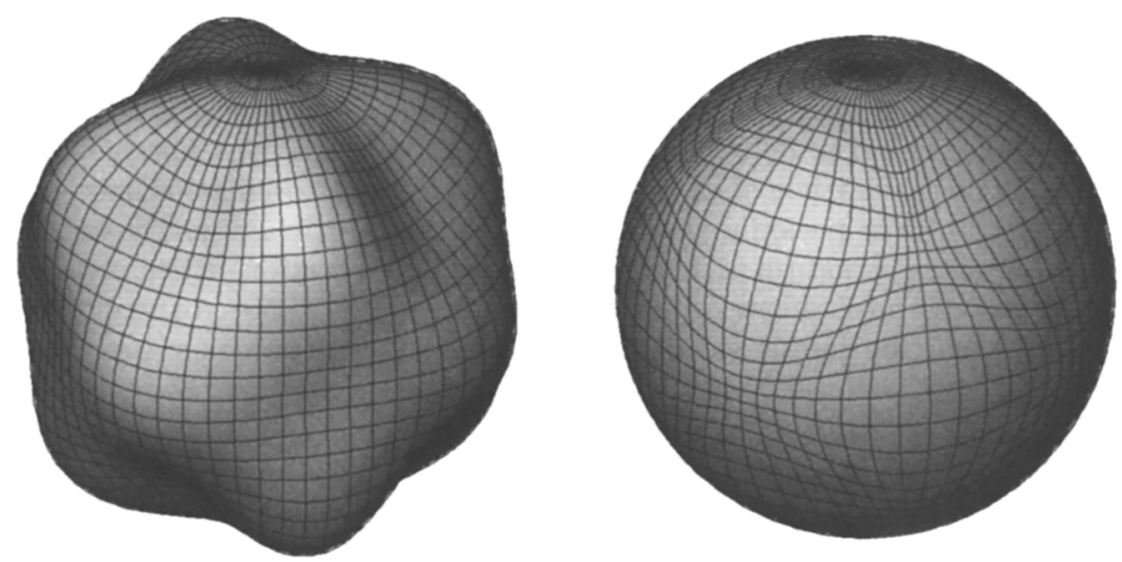

Figure 1. A star distorted by $m=-4$ modes of frequencies $\omega=10 \omega_{d}$ (left) and $\omega=0.1 \omega_{d}$ (right), corresponding to $\mathrm{p}$ and $\mathrm{g}$ modes, respectively. The lines indicate the nominal boundaries of photospheric fluid elements; without the pulsation, they would reduce to latitude and longitude arcs. In this and the subsequent figures, I've deliberately exaggerated the amplitude of pulsation to highlight the effects caused by it.

g mode. Since the azimuthal order is negative for these modes, the distortions caused by the pulsation would propagate in the prograde direction - that is, from left to right, the figures having been oriented with the north pole at the top. The grid lines drawn on the stellar surface, indicating the nominal boundaries of photospheric fluid elements, clearly indicate the principal difference between $\mathrm{p}$ and $\mathrm{g}$ modes: the former are dominated by radial fluid motions, and the latter by tangential ones.

\section{Inertial Forces}

When a pulsating star is gradually spun up from the non-rotating situation, the zeroth-order effect of the rotation (assumed throughout to be rigid) is simply to carry the pulsation along with it, much like ocean waves are carried around by the Earth's rotation. This means that, when seen from an inertial frame of reference, the frequency of pulsation becomes Doppler shifted to

$$
\sigma=\omega-m \Omega
$$

where $\Omega$ is the rotation angular frequency of the star. From this expression, we can see that positive- $m$ modes (which would appear retrograde in the co-rotating reference frame) can appear prograde in an inertial frame (i.e., $-\sigma / m>0$ ), if $\Omega$ is sufficiently large that $\sigma$ becomes negative.

As the rotation becomes more rapid, the centrifugal and Coriolis forces will begin to have an effect on the pulsation. These fictitious forces arise due to the non-inertial nature of the co-rotating frame in which waves are carried; the following subsections consider each in turn. 


\subsection{The Centrifugal Force}

In a star rotating as a rigid body, the centrifugal force behaves much like the gravitational force, and we may combine the two to define an effective gravity at each point. This effective gravity is not spherically symmetric; at a given radius within the star, it is stronger at lower latitudes, and - save at equator and poles - it is not directed towards the stellar centre. Therefore, the configuration of the star becomes distorted relative to the non-rotating case, with an extension of the equatorial regions and a (lesser) flattening of the poles.

It is through this distortion that the centrifugal force exerts a proxy influence on pulsation; there can also be secondary effects, arising from direct interactions between the pulsation and the effective gravitational potential, but in most cases these are small enough that they can safely be ignored. The degree of the distortion scales with $\Omega^{2}$, and its effect on pulsation will be most important in those stars rotating at a significant fraction of the critical rotation frequency

$$
\Omega_{\mathrm{c}}=\sqrt{\frac{8 G M}{27 R^{3}}}=\sqrt{\frac{8}{27}} \omega_{\mathrm{d}}
$$

at which centrifugally-assisted mass loss from the distended stellar equator would commence (here, $R$ now refers to the polar radius of the distorted star).

The past decade has seen significant progress towards incorporating the centrifugal force in pulsation calculations (e.g. Lee \& Baraffe 1995; Soufi, Goupil \& Dziembowski 1998; Lignières \& Rieutord, these proceedings). However, most studies are still limited to ad-hoc or incomplete models for the distorted equilibrium configuration of the star, which fall well short of the current levels of sophistication found in stellar evolution theory (e.g. Maeder, these proceedings). Hopefully, new calculations based around physically-realistic models will be attempted in the very near future.

\subsection{The Coriolis Force}

In terms of its effect on pulsation, the Coriolis force is quite different from its centrifugal counterpart - and, in my own opinion, much more interesting. For a fluid element moving with velocity $\mathbf{v}$ in the frame co-rotating with vectorial angular velocity $\boldsymbol{\Omega}$ (such that $|\boldsymbol{\Omega}|=\Omega$ ), this force is given by

$$
\mathbf{F}_{\mathrm{c}}=2 \mathbf{v} \times \boldsymbol{\Omega} .
$$

The appearance of the fluid velocity $\mathbf{v}$ in this expression is particularly significant: it produces an extra term in the governing pulsation equations, which can significantly modify the gross qualitative character of waves within the star. Contrast this with the centrifugal effect, which manifests itself primarily via modifications to the underlying medium through which the waves propagate, leaving the wave equations themselves unaffected.

To determine the importance of the Coriolis force to the propagation of a given wave, we can take a leaf out of any book on atmospheric physics (e.g. Gill 1982), and compare its wavelength $\lambda$ to the so-called Rossby radius

$$
R_{\mathrm{r}}=\left|\frac{c}{2 \Omega}\right| ;
$$


here, as before, $c$ is the wave's phase velocity. The Rossby radius, a measure of how far a wave propagates during one rotation period, is the fundamental length scale over which the Coriolis force makes itself felt. If $\lambda$ is longer than this scale (i.e, $\lambda>R_{\mathrm{r}}$ ), then a wave's dynamics are dominated by the Coriolis force; conversely, if $\lambda<R_{\mathrm{r}}$, the force will have little effect. This is the reason why, contrary to urban legend, the Coriolis force has a negligible effect on the draining of water from a bathtub: $R_{\mathrm{r}}$ is typically a couple of kilometres for such a system, orders of magnitude longer than the length of the tub. A toy rubber duck would have more influence on the outflow!

Focusing now on the case of stellar pulsation, recall that the azimuthal wavelength and phase velocity of a mode are given by $\lambda=1 / m$ and $c=-\omega / m$, respectively. Accordingly, we can easily show from the foregoing discussion that the Coriolis force will dominate the mode's dynamics whenever

$$
\frac{2 \Omega}{\omega}>1,
$$

a situation sometimes referred to as the 'inertial regime'. Since $\Omega$ must always be smaller than the critical frequency $\Omega_{\mathrm{c}}$ (cf. Eq. 4), it is clear that the Coriolis force is important to those modes whose frequencies satisfy

$$
\omega<2 \sqrt{\frac{8}{27}} \omega_{\mathrm{d}} \approx \omega_{\mathrm{d}}
$$

Therefore, we can see that the p modes (for which $\omega>\omega_{\mathrm{d}}$ ) will remain relatively unaffected by the Coriolis force; only the low-frequency g modes will be appreciably influenced by it. Precisely what this influence is will be considered in the following section.

\section{The Equatorial Waveguide}

To provide a relatively simple demonstration of the action of the Coriolis force, we can look at its effect on a $m=-4$ prograde sectoral g mode (here, 'sectoral' refers to the fact that the phase of perturbations is constant along lines of longitude). Figure 2, which shows both the surface distortion and fluid velocities of a non-rotating star perturbed by this mode, demonstrates that the motions of fluid elements are predominantly in the azimuthal direction. Eq. (5) then tells use that the Coriolis force arising from these motions will have components in both radial and polar directions. At sufficiently low frequencies (i.e., $\omega \ll \omega_{\mathrm{d}}$ ), the radial component can be ignored, since buoyancy will be far more important in governing the (almost-negligible) radial motion of elements. This leaves the polar component, which for azimuthal fluid velocity $v_{\phi}$ will be given by

$$
F_{\mathrm{c}, \theta}=2 v_{\phi} \Omega \cos \theta .
$$

Now, in the case of the $m=-4$ sectoral g mode shown in Fig. 2, $v_{\phi}$ is large and positive for those dense fluid elements situated along loci of maximum compression (one such locus can be seen slightly to the right of disk-centre, running in a north-south direction). The above expression indicates that the polar 

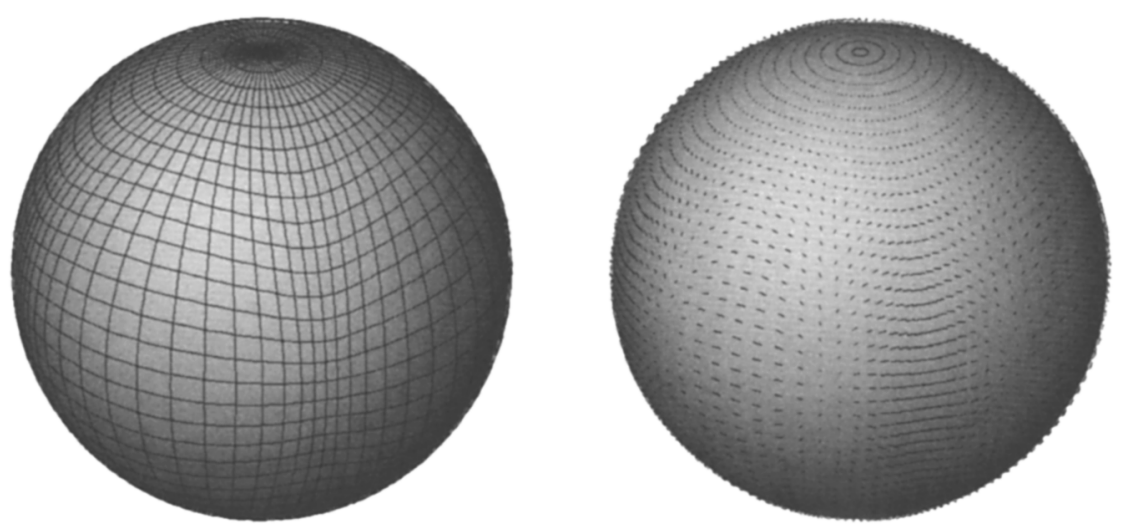

Figure 2. The distortion (left) and velocity fields (right) of the surface of a non-rotating star subject to an $m=-4$ prograde sectoral g mode; the latter are shown using tick marks, whose lengths are proportional to the local fluid velocity.

Coriolis force on these elements will be directed towards the equator - no matter whether the elements are situated in the northern or southern hemisphere. This equator-ward pull will tend to enhance the compression near the stellar equator.

Likewise, for the rarefied fluid elements located along loci of maximum expansion, $v_{\phi}$ is large and negative, and the Coriolis force will be directed towards the poles. The pole-ward pull tends to reduce the compression near the stellar equator, and thereby increase the expansion. The net effect of both processes is that the pulsation-originated perturbations around the equator - be they compression or expansion - become enhanced, at the expense of those at higher latitudes. It is as if the pulsation has become confined within a waveguide, which restricts the extent of perturbations to a band around the stellar equator.

Figure 3 provides an example of trapping within this equatorial waveguide; it shows the surface distortion and fluid velocities of the same $m=-4$ sectoral g mode considered in Fig. 2, but with the added influence of the Coriolis force (calculated assuming that $2 \Omega / \omega=10$ ). Clearly, perturbations are now restricted to the equatorial regions, with higher latitudes $\left(> \pm 15^{\circ}\right)$ of the star remaining relatively unaffected by the pulsation.

In principle, trapping within an equatorial waveguide can happen to any mode, provided that the inertial regime (cf. Eq. 7) is reached; I've focused here on a prograde sectoral g mode simply to provide the clearest illustration of the physics. The phenomenon was discovered in the context of terrestrial gravity waves by Matsuno (1966); however, only relatively recently has its relevance to stellar pulsation been recognized (see Lee \& Saio 1990; Bildsten, Ushomirsky \& Cutler 1997). As demonstrated by the latter authors, the approximate location of the waveguide boundaries can be estimated from

$$
|\cos \theta| \approx \frac{\omega}{2 \Omega}
$$



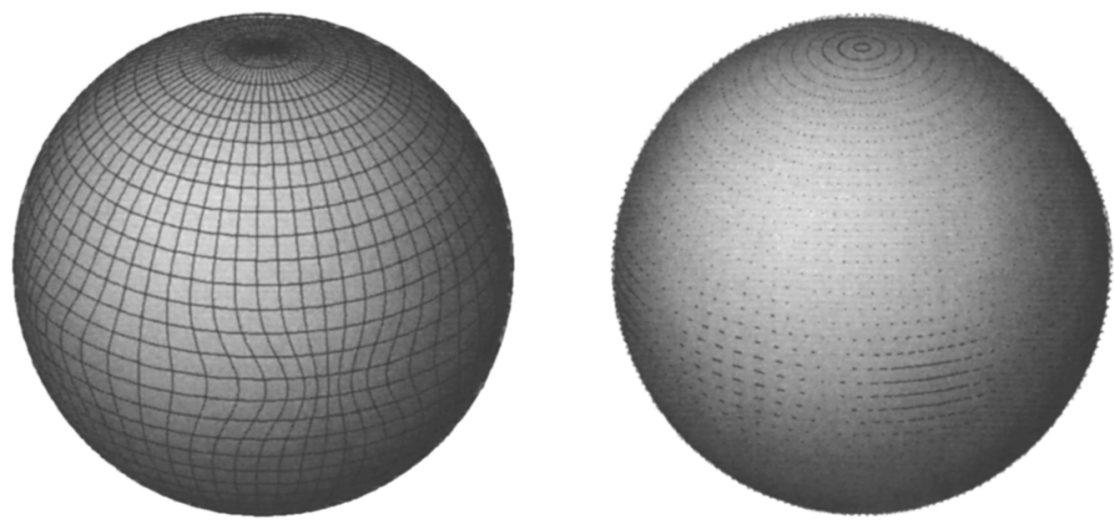

Figure 3. The distortion (left) and velocity fields (right) of the surface of a rotating star subject to an $m=-4$ prograde sectoral $\mathrm{g}$ mode; compare with Fig. 2, noting the equatorial confinement caused by the Coriolis force.

which tells us that, towards more-rapid rotation, the waveguide will become ever-narrower.

Equatorial trapping may be an important part of the (currently-uncertain) mechanism by which Be stars form their circumstellar disks. It provides a means of focusing pulsation kinetic energy towards the equatorial regions of a star, enhancing the velocity perturbations in these regions relative to the non-rotating case. For a star spinning sufficiently close to its critical rate (cf. Eq. 4), the extra kick provided by the velocity enhancements may be sufficient to launch material into orbit (see Owocki, these proceedings).

Trapping can also have ramifications for those attempting to observe pulsation in rapidly-rotating stars. Since the perturbations caused by a trapped mode cover only a small fraction of the stellar surface, the associated light variations can be very difficult to detect (Townsend 2003). This may explain why recent photometric observations of open clusters, for which the majority of stars exhibit large $v \sin i$ values, have failed to find any significant evidence for low-frequency g-mode pulsation (see e.g. Balona 1994).

\section{Rossby Waves}

In the preceding section, I demonstrated how the Coriolis force can trap pulsation within an equatorial waveguide. However, this is not the only trick up its sleeve; it can also generate wholly-new types of wave, which do not exist in non-rotating stars. If we consider fluid motions confined to a stably-stratified layer of a rotating star, it can be shown (e.g. Unno et al. 1989) that they must satisfy

$$
\frac{\mathrm{d}}{\mathrm{d} t}(\zeta+2 \Omega \cos \theta)=0
$$

in the co-rotating frame; here, $\zeta$ is the radial component of the vorticity, which measures the rate at which fluid elements are rotating around an axis perpen- 

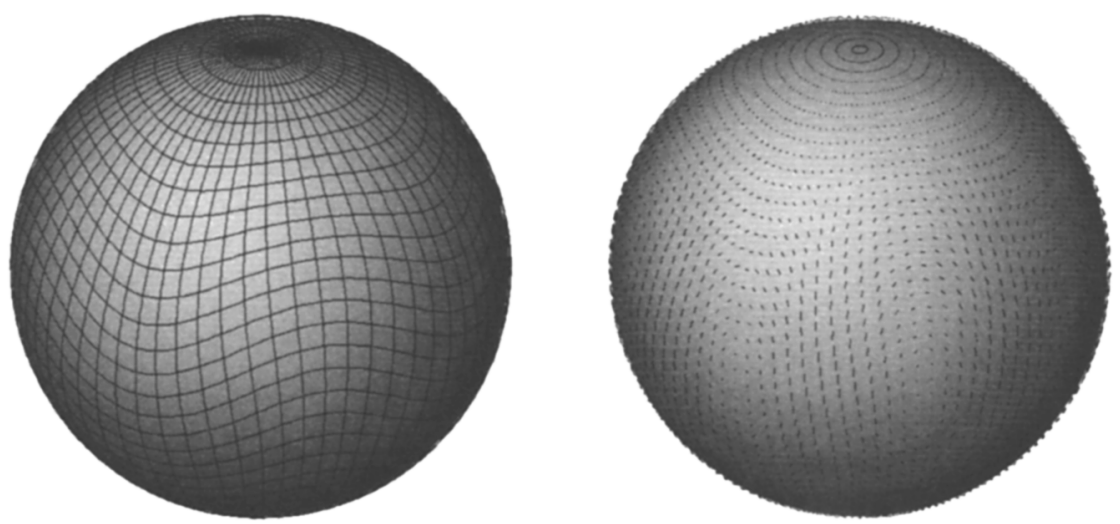

Figure 4. The distortion (left) and velocity fields (right) of the surface of a rotating star subject to an $m=4$ Rossby mode.

dicular to the layer. This equation is a conservation law for angular momentum - it tells us that any changes to the polar position $\theta$ of a fluid element must be exactly matched by corresponding changes to $\zeta$. Specifically, if the element (initially at rest, such that $\zeta=0$ ) is subject to a perturbation which moves it northwards, then $\zeta$ will become negative, and the element will start to rotate in a clockwise direction, as seen from above the layer. Conversely, a southwards motion will cause $\zeta$ to become positive, associated with anti-clockwise rotation.

Since the fluid element does not exist in isolation, any vorticity arising from its north-south movement will influence the neighbouring fluid elements, causing them to undergo motions themselves, and - via the conservation law (11) - acquire a vorticity of their own. This transfer will cascade around the star, amounting to the propagation of a wave. A more detailed analysis (e.g. Saio 1982) demonstrates that these Rossby waves (sometimes known as $\mathrm{r}$ modes) will always propagate in a retrograde direction, as seen in the co-rotating frame.

Figure 4 shows the surface distortion and fluid velocities caused an $m=4$ Rossby wave. The figure demonstrates two hallmark characteristics of these waves. Firstly, velocity streamlines take the form of large whorls on the stellar surface. Secondly, even though the shapes of each perturbed fluid element differ, their sizes remain approximately constant, indicating that the star is behaving as if it were incompressible. The latter fact means that Rossby waves do not produce local temperature variations, making them impossible to detect from photometric observations alone. The absence of temperature variations also inhibits the internal excitation of Rossby waves; some external source of energy (e.g. torque from a binary companion) must exist in order for them to be excited.

\section{Frequency Splitting}

So far, I've discussed the effect of rotation on the spatial structure of pulsation, but rotation can also influence its corresponding frequency spectrum. In a nonrotating star, certain multiplets of modes, sharing the same radial-polar depen- 

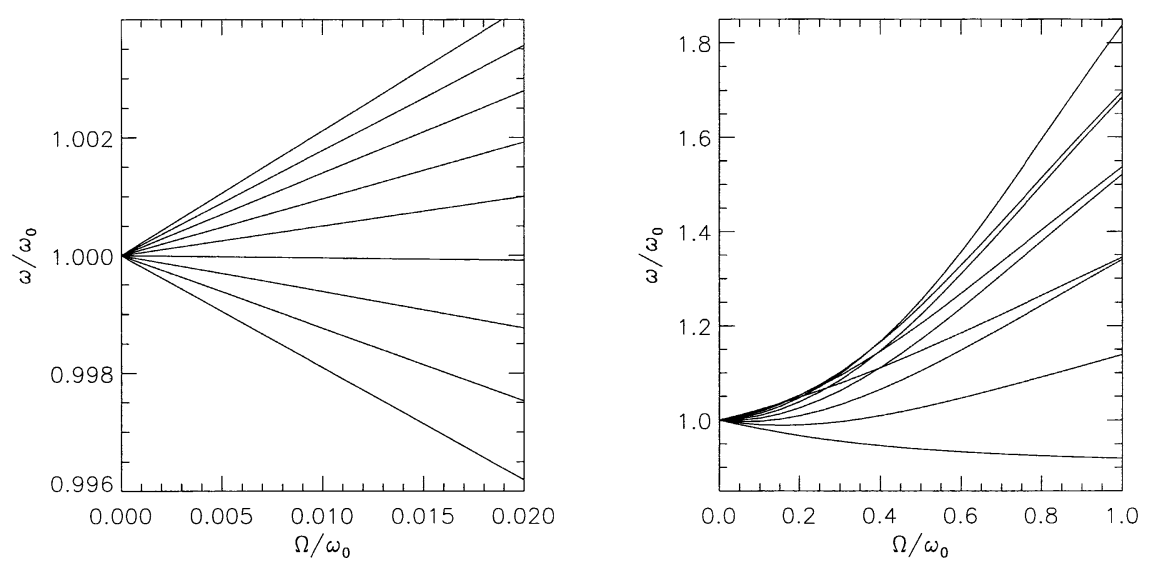

Figure 5. The pulsation frequencies $\omega$ of an $m=-4 \ldots 4$ noniplet of modes, plotted as a function of rotation frequency $\Omega$. The right-hand panel shows the same data as the left-hand one, but over an increased range of $\Omega$. Both ordinate and abscissa are normalized by the frequency $\omega_{0}$ obtaining in the non-rotating limit.

dence $f_{y}(r, \theta)$ but having differing azimuthal orders (cf. Eq. 1), are degenerate in pulsation frequency. However, this degeneracy is lifted with the introduction of rotation. As demonstrated in the seminal paper by Ledoux (1951), the multiplet splitting is uniform in $m$ when the rotation is sufficiently slow, and may be described by

$$
\omega=\omega_{0}+m \Omega C,
$$

where $\omega_{0}$ is the frequency of the multiplet in the non-rotating limit, and the Ledoux constant $C$ depends on the multiplet in question.

The left-hand panel of Fig. 5 gives an example of the uniform splitting described above; the frequencies of a noniplet, with azimuthal orders ranging in integer steps from $m=-4$ to $m=4$, are plotted as a function of $\Omega$. The structure shown is reminiscent of the Zeeman effect of atomic physics, and much like we can infer magnetic field strengths from spectroscopy - it is possible to determine the rotation frequency $\Omega$ of a star by measuring the frequency spacings of its pulsation multiplets (see e.g. Aerts, these proceedings). Needless to say, this requires that the multiplet first be correctly identified, allowing us to calculate and eliminate $C$.

Eq. (12) is correct to first order in $\Omega$, and as such includes only the effects of the Coriolis force. As the rotation becomes more rapid, second- and higher-order terms, arising from both centrifugal and Coriolis forces, become important in determining the degree of splitting. This is illustrated in the right-hand panel Fig. 5, which is a continuation of its left-hand counterpart to larger rotation frequencies. Clearly, the splitting becomes highly non-uniform as $\Omega$ approaches similar values to $\omega_{0}$, and in such circumstances it becomes quite difficult to ascertain whether a group of observed frequencies do indeed belong to the same multiplet. 


\section{Angular-Momentum Transfer}

I've placed the focus throughout the paper on the effect which rotation can have on pulsation. However, the interaction between the two is by no means a oneway street: pulsation can also exert an influence over rotation. This is because, like all wave phenomena, pulsation modes are capable of transporting angular momentum. Ando (1983, and references therein) considered whether such transport is significant, in terms of the rotational evolution of the early-type $\beta$ Ceph and 53 Per classes of pulsating star. He found that the timescale for complete angular momentum redistribution within such stars is significantly shorter than their main-sequence lifetimes. This is a startling result, since it implies that studies of stellar rotation which neglect pulsation-facilitated redistribution (as almost all do, although see Talon \& Charbonnel, these proceedings) could be in serious error.

I should stress that Ando was forced to make estimates regarding the character of $\mathrm{p}$ and $\mathrm{g}$ modes in his stellar models, since prior to the 1990s it was not clear what excitation mechanism was responsible for the pulsation of earlytype stars. A follow-up investigation, based on more-recent data, is therefore required to determine whether his findings remain valid. My own prejudice is that they most likely do, and I therefore feel that it is appropriate to close with a quote from the abstract of Ando's paper: 'We conclude that the wave-rotation interaction cannot be disregarded in astrophysical problems'.

\section{Animations}

I've prepared computer animations of figs. 1-4, which may be downloaded from my website (http://www.star.ucl.ac.uk/ rhdt/). I urge interested readers to take a look at these, since they illustrate - much better than my figures - many of the phenomena I have discussed in the paper.

\section{References}

Ando, H. 1983, PASJ35, 343

Balona, L. A. 1994, MNRAS267, 1060

Bildsten, L., Ushomirsky, G., Cutler, C. 1996, ApJ460, 827

Dziembowski, W. A., Pamyatnykh, A. A. 1993, MNRAS262, 204

Gill, A. E. 1982, Atmosphere-Ocean Dynamics. Academic Press, London

Ledoux, P. 1951, ApJ114, 373

Lee, U., Baraffe, I. 1995, A\&A301, 419

Lee, U., Saio, H. 1990, ApJ349, 570

Matsuno, T. 1966, J. Meteor. Soc. Japan, 44, 25

Saio, H. 1982, ApJ256, 717

Soufi, F., Goupil, M. J., Dziembowski, W. A. 1998, A\&A334, 911

Townsend, R. H. D. 2003, MNRASin press

Unno, W., Osaki, Y., Ando, H., Saio, H., Shibahashi, H. 1989, Nonradial Oscillations of Stars, 2nd edn. University of Tokyo Press, Tokyo 